\title{
Simulation and Experiment on Electric Field Emissions Generated by Wireless Energy Transfer
}

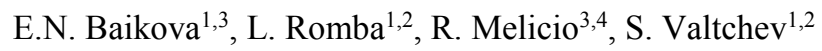 \\ ${ }^{1}$ UNINOVA-CTS and ${ }^{2}$ FCT of Universidade Nova de Lisboa \\ ${ }^{3}$ EST Setúbal, Instituto Politécnico de Setúbal, Setúbal \\ ${ }^{4} \mathrm{ICT}$, Universidade de Évora, Escola de Ciências e Tecnologia, Departamento de Física \\ 5IDMEC, Instituto Superior Técnico, Universidade de Lisboa (ruimelicio@gmail.com)
}

\begin{abstract}
This paper presents a wireless energy transfer (WET) system operating at the frequency of tens of $\mathrm{kHz}$. It treats the modeling and simulation of WET prototype and its comparison with experimental measuring results. The wireless energy transfer system model was created to simulate the electric field between the emitting and the receiving coils, applying the finite element method. The results from the simulation are compared to the measured values of the electric field emission from the wireless energy transfer equipment. In the recent years the interest in the WET technology, especially for the electric vehicles (EV) batteries charging, is rapidly growing. The WET systems pollute the environment by electromagnetic emissions. Due to the expanding use of this technology in industrial and consumer electronics products, the problems associated with the electromagnetic compatibility (EMC), and the adverse impact on the human health becomes highly important.
\end{abstract}

Keywords: wireless energy transfer, electric field, modeling, simulation, prototype.

\section{Introduction}

The attention to the electric transportation system is aimed to the improvement of the safety, including the safer battery charging, e.g. by contactless energy transfer [13]. In the last decade, the magnetic resonance technology is considered to be the most suitable for the contactless EV battery charging $[2,4,5]$. It does not require an accurately parking of the vehicle, being not so sensitive to the alignment $[4,5]$.

However, the WET systems represent the EMC problems. The electromagnetic field (EMF), through which the energy is transferred between the emitting coil and the receiving coil, is a high-frequency field and induces electromagnetic interferences (EMI) into the other electronic devices. This EMF has high intensity and so may influence the operation of the communication channel by which the emitter and the receiver exchange the information $[4,6]$. Moreover, the electromagnetic field emitted by the WET system can induce high voltages and currents in the living organisms [48]. Thus, one of the fundamental concerns for the WET development is the conformity with EMF safety regulations and standards [9-10]. 
The EMC issue of the WET system requires a detailed analysis of the EM processes in the living organisms exposed to EMF. A possibility to estimate the EMF impact on the living beings is by modeling and simulation. Recently, some papers were published, discussing the modeling and simulation of the electromagnetic process in WET systems [8, 11-12].

In [8], a dosimetric numerical study is presented, assessing the exposure of the human in a proximity of a wireless EV battery charging prototype. The prototype is delivering a power of $560 \mathrm{~W}$ at operating frequency $85 \mathrm{kHz}$. The electric field induced in a homogeneous ellipsoid phantom and in an anatomical model of human body, exposed to a WET system prototype was evaluated.

In [11], the magnetic field from the WET system for realistic EV charging at a power level of $7 \mathrm{~kW}$, is modeled and simulated using the Ansys Maxwell software. To validate the model, the simulated magnetic field is compared to the measured one. Finally, the electric field induced in a human body model by a leaked magnetic field from a WET system, is evaluated.

In [12], the scenarios of multiple WET systems are considered, working in parallel simultaneously. Using the Ansys Maxwell software and finite element method (FEM) the electric field and voltage, induced in the human body by the leakage EMF, are simulated and evaluated. The specific absorption rate in specific tissues of the human body, positioned at various orientations, are also simulated and assessed. The simulation results are compared to the basic margins defined in the international regulations, determined as boundaries for the human exposure to time-varying EMFs.

In the existing research papers on a WET system, more attention is dedicated to the magnetic field, which is logical having in mind that the transport of energy is made by the magnetic field. Few works deal with simulating and measuring of the electric field produced by WET systems. However, the coupling mechanisms of the EMF components, i.e. electric and magnetic fields, are different. Thus, for a more complete characterizing of the human exposure, both components must be simulated and measured [13].

In this paper, the modeling and simulation of the WET system operating at the frequency of $20 \mathrm{kHz}$, is presented. The results of electric field simulation are compared to the measurements of the electric field emissions radiated by a prototype of the WET system. The simulation and measurement results are compared to the international EMF safety standards and regulations.

\section{Technological Innovation for Resilient Systems}

The concept of system resilience is important and hyper-popular over the last few years [14]. It implies that the systems should be able to give safety and reliability, keeping the capability to act to sustain and rectify the infrastructure components without disturbing the service [15]. Some definitions of resilience comprise requirements on the recovery of the system, safety, including the safety of the humans, some parts of security and reliability of the system [16].

In the recent years, the market for EV is growing steadily. Most of EV battery supply today are unidirectional, i.e. they can only store electricity by charging the 
battery. Correspondingly, most of EV charging stations are designed for unidirectional electricity delivery to the vehicle [17]. With an increased infrastructure and market demand on the electric grid, the bidirectional, i.e. V2G-capable, EVs should be able to deliver energy from their batteries to the grid via bidirectional charging stations by using WET systems. The purpose of the smart grids is to reduce the energy costs, and in the same time, to reach a sustainable balance between the production and consumption, increasing the resiliency of the grid. Thus, using WET systems, EVs can improve the sustainability and resiliency of the power grids and the quality of electrical energy transferred to the customers [17].

On the other hand, as WET technology is becoming more extensively applied in high power applications, the human exposure to EMFs consequently increases [12]. Moreover, the electronic components of infrastructure may be impacted by the presence of oscillating EMFs associated with a WET system. This means that the WET system may become a source of hazard for human health and electronic equipment and, this way, reduce the infrastructure resilience. So, to attenuate the negative impact of the EM fields from wireless EV charging system and to increase the system resilience, the mitigation strategies should be employed. In [18,19] some techniques for the reduction of the EMFs produced by wireless EV charging systems are proposed, applying various shielding methods, e.g. conductive, magnetic, active shield and reactive shield coil. By applying those shielding methods, the general resilience will be improved.

\section{Modeling}

The finite element method has been chosen for modeling of the WET system. A frequency-domain analysis was applied to evaluate the electric field distribution in the WET surrounding area.

The electromagnetic phenomena in the WET system are governed by the Maxwell equations, which are given below in their differential form [20]:

$$
\begin{gathered}
\nabla \times \mathbf{E}=-\frac{\partial \mathbf{B}}{\partial t} \\
\nabla \times \mathbf{H}=\mathbf{J}+\frac{\partial \mathbf{D}}{\partial t} \\
\nabla \cdot \mathbf{D}=\rho \\
\nabla \cdot \mathbf{B}=0
\end{gathered}
$$

The equation of continuity is given by:

$$
\nabla \cdot \mathbf{J}=-\frac{\partial \rho}{\partial t}
$$

where the vectors $\mathbf{E}$ is the electric field, $\mathbf{H}$ is the magnetic field, $\mathbf{D}$ is electric displacement field, $\mathbf{B}$ is magnetic flux density and $\mathbf{J}$ is conducting current density. 
The following group of constitutive equations connects the field vectors with the environment parameters:

$$
\mathbf{B}=\mu_{0} \mu_{r} \mathbf{H} \quad \mathbf{D}=\varepsilon_{0} \varepsilon_{r} \mathbf{E} \quad \mathbf{J}=\sigma . \mathbf{E}
$$

where $\mu_{0}$ and $\varepsilon_{0}$ are magnetic permeability and electric permittivity of the free space, respectively, $\mu_{\mathrm{r}}$ and $\varepsilon_{\mathrm{r}}$ are the relative permittivity and relative permeability of a medium, respectively, $\sigma$ is the electric conductivity.

In the quasi-static approximation, the displacement currents defined by $\mathbf{D}$ are negligible, so the Maxwell equations (1) and (2) in the frequency domain are given by:

$$
\begin{gathered}
\nabla \times \mathbf{H}=\mathbf{J} \\
\nabla \times \mathbf{E}=-j \omega \mathbf{B}
\end{gathered}
$$

The magnetic flux density $\mathbf{B}$ in terms of the magnetic vector potential $\mathbf{A}$ is given by:

$$
\mathbf{B}=\nabla \times \mathbf{A}
$$

Combining the equations (1) to (9) gives the equation (10), from where the magnetic vector potential A can be determined:

$$
\nabla \times\left(\frac{1}{\mu} \cdot \nabla \times \mathbf{A}\right)+j \omega \sigma \mathbf{A}=\mathbf{J}
$$

Next, the magnetic field distribution is obtained using (9). Lastly, the electric field is calculated in accordance with the constitutive equations (6) and the Ampere law (7).

\section{Proposed Design and Simulation}

The proposed design of the WET system model including coils, ferrite cores, aluminum profiles and surrounding (air) domain, has been simulated using the numerical software. Due to the symmetry of the WET system, $1 / 4$ of the full structure, which is shown in Fig. 1, was modeled and simulated. The model used for the electromagnetic simulation with size in mm shown in Fig. 1.
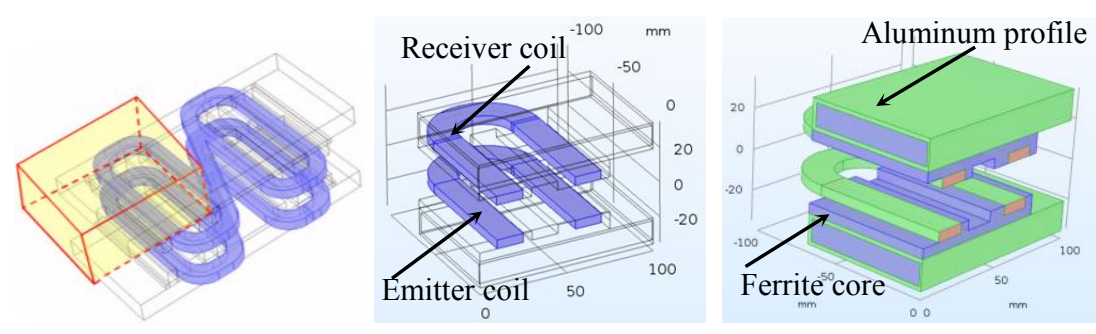

Fig. 1. Modelling set of ferrite cores, aluminum profiles and inductive coils. 
The WET charger system consists of ferrite cores for the emitter and receiver coils made of copper with the section of $1 \mathrm{~mm}^{2}$ and of aluminum profile. The number of turns in the emitter coil is $\mathrm{N}_{\mathrm{e}}=36$ and in the receiver coil is $\mathrm{N}_{\mathrm{r}}=4$. The simulation was performed by applying to the WET system the first harmonic of the rectangular voltage waveform, neglecting the higher order harmonics.

The magnitudes of the electric field from $262 \mathrm{~W}$ WET system were simulated for the air gap between the emitter and the receiver coils of $20 \mathrm{~mm}$. The electric field distribution for $20 \mathrm{kHz}$ frequency range with contours lines, which represent the magnitude of the electric field, is shown in Fig. 2. The bar on the right is illustrating the values of E-field $(\mathrm{V} / \mathrm{m})$ in certain parts of the model.

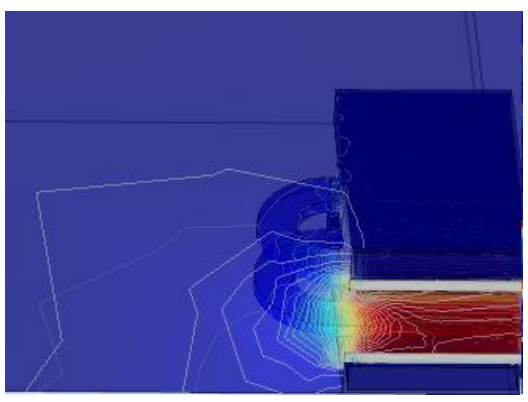

a)

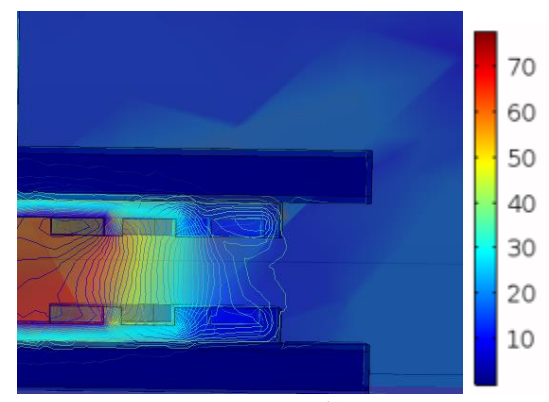

b)

Fig. 2. WET system E-field generated at the air gap of: a) side view; b) cross-section view.

According to the International Commission on Non-Ionizing Radiation Protection (as) Guideline, the reference level for general public exposure to time-varying electric field for the frequency range of $3 \mathrm{kHz}$ to $10 \mathrm{MHz}$ is $83 \mathrm{~V} / \mathrm{m} \mathrm{[9]}$. As it is shown in Fig. 2, the electric field intensity is below the exposure limits defined by ICNIRP. However, in the regions limited by the ferrite cores and aluminum profiles the value of electric field reaches $75 \mathrm{~V} / \mathrm{m}$, i.e. is close to this limit. These results also confirm that the strength of the electric field decreases rapidly with the distance from the WET system and reaches $5.72 \mathrm{~V} / \mathrm{m}$ at the distance of $200 \mathrm{~mm}$.

From the Fig. 2 it can be observed that the ferrite core and aluminum profile are effectively confining and guiding the EMF.

\section{Experimental Setup}

The model with the given setup and specifications was used for an experimental verification of the results of a previous research [21,22]. The equipment for the measurement of the electric field was composed by a WET power converter and a measuring instrument Narda SRM-3000 (Selective Radiation Meter). The analysis is focused on the measured emission of electric field, from the WET prototype, operating at $20 \mathrm{kHz}$ frequency. The block diagram of the studied WET system for EV charging is shown in Fig. 3.

The emitter coil with the ferrite core, aluminum profile and experimental setup of the WET prototype is shown in Fig. 4. 


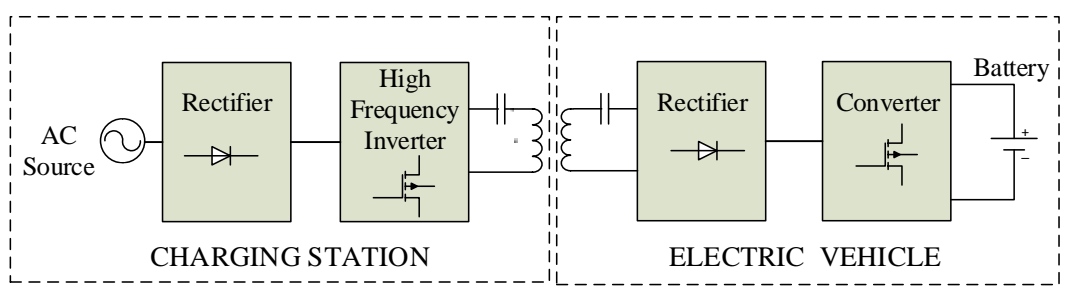

Fig. 3. Block diagram of the WET system for EV battery charging
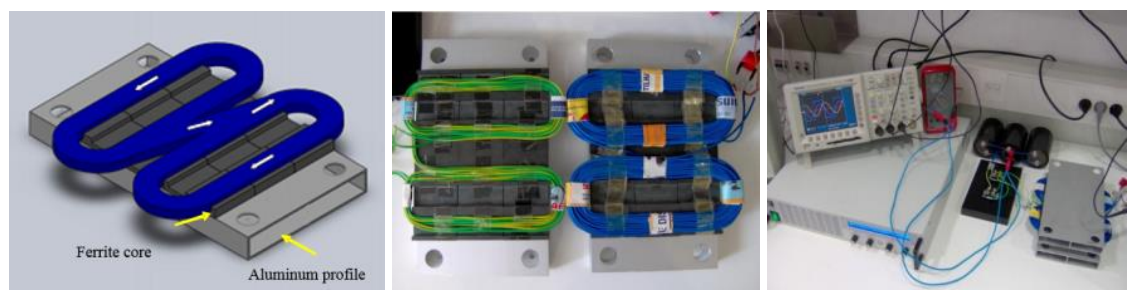

Fig. 4. 3D vision of emitter coil and experimental setup of the WET prototype.

Applying the Fourier series expansion, the square wave AC input which supplies the emitter circuit can be transformed into a sum of odd harmonics. So, to estimate the electromagnetic interference impact from the WET prototype with output power value of $262 \mathrm{~W}$, the RMS values of harmonics generated by the WET system with an air gap of $20 \mathrm{~mm}$ were measured.

The used measuring equipment was able to evaluate frequencies starting from $100 \mathrm{kHz}$. In this way, the amplitudes of the harmonics were measured from the 5 th harmonic, whose frequency corresponds to $100 \mathrm{kHz}$. The minimum squares method was used to obtain the 1 st and the 3rd harmonics amplitudes. According to this method, the experimental data were attuned with a coefficient of determination $R^{2}=0.9999$. The high value of the coefficient of determination shows that the trend line is quite exactly consistent with the data. The amplitudes of the 25 odd harmonics, including the 1 st and the 3 rd ones calculated by minimum squares method, is shown in Fig. 5.

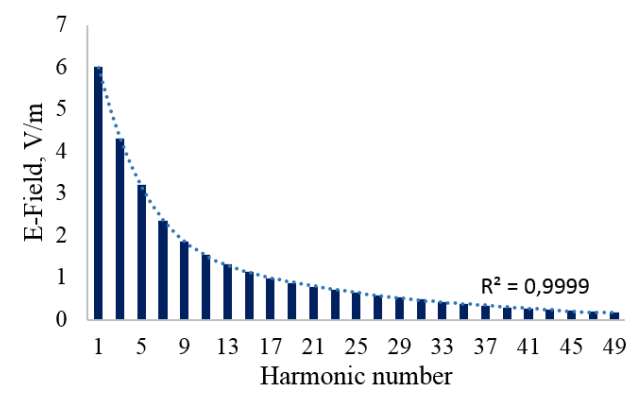

Fig. 5. Odd harmonics amplitudes generated by the WET prototype. 
According to ICNIRP Guidelines [9] for situations with simultaneous exposures to fields of multiple frequencies, these exposures have a cumulative effect. In this case, the following criteria [9] must be applied:

$$
\sum_{j=1 H z}^{10 M H z} E_{j} / E_{R, j} \leq 1
$$

where $E_{j}$ is the electric field strength at frequency $j, E_{R, j}$ is the reference level of electric field strength at frequency $j$ according to [9].

The total level of electric field radiated by WET prototype operating at $20 \mathrm{kHz}$ frequency is $E_{j}=29.71 \mathrm{~V} / \mathrm{m}$ and $E_{R, j}=83 \mathrm{~V} / \mathrm{m}$. Applying (11) the criteria value is 0.36 , i.e. below 1 . Thus, this result confirmed that the level of the electric field generated by WET prototype is below the exposure limits defined by ICNIRP [9].

By comparing the simulated and measured results of the electric field 1st harmonic, it can be concluded that the simulation results are in agreement with the measured data: the simulated electric field $-5.62 \mathrm{~V} / \mathrm{m}$ and the measured electric field $-6.03 \mathrm{~V} / \mathrm{m}$. The WET system considered in this research, in fact, is not a linear system. Thus, the discrepancy between simulated and measured results can be explained by the linearity assumptions taken in the computational simulation.

\section{Conclusions}

The WET system described here is modeled and simulated at the frequency of $20 \mathrm{kHz}$. The electric field density was simulated at an air gap of $20 \mathrm{~mm}$ between the emitter and the receiver, and then measurements were executed at the same physical and geometrical conditions. The simulated electric field values are compared to the measured ones. The comparison shows an error of roughly $6.7 \%$ between the simulation and the reality. The present study is useful for a more complete knowledge about the EMI, not only about the magnetic field component produced by the WET system, but also to take a deeper view into the influence of the electric field on the human health.

\section{References}

1. J.T. Boys, G.A. Covic, A.W. Green: Stability and control of inductively coupled power transfer systems, IEE Proceed. Electric Power Applications, vol. 147(1), pp. 37-43 (2002)

$2 \mathrm{~S}$. Li, C. Mi, C.: Wireless power transfer for electric vehicle applications, IEEE Journal of Emerging and Selected Topics in Power Electronics, vol. 3(1), pp. 4-17 (2015)

3. T. Imura, H. Okabe, Y. Hori: Basic experimental study on helical antennas of wireless power transfer for electric vehicles by using magnetic resonant couplings, Proc. IEEE Vehicle Power and Propulsion Conference, pp. 936-940, Dearborn, USA (2009)

4 S. Kim, H.-H. Park, J. Kim, K. Kim, S. Ahn: Design and analysis of a resonant reactive shield for a wireless power electric vehicle, IEEE Transactions on Microwave Theory and Techniques, vol. 62(4), pp. 1057-1066 (2014) 
5. Y. Gao, K. B. Farley, A. Ginart, Z.T.H. Tse: Safety and efficiency of the wireless charging of electric vehicles, Proc. of the Institution of Mechanical Engineers, Part D: Journal of Automobile Engineering, vol. 230(9), pp. 1196-1207 (2015)

6. E.N. Baikova, S.S. Valtchev, R. Melicio, A. Krusteva, V. Fernão Pires: Study of the electromagnetic interference generated by wireless power transfer systems, International Review of Electrical Engineering, vol. 11(5), pp. 526-534 (2016)

7. A. Christ, M.G. Douglas, J.M. Roman, E.B. Cooper, A.P. Sample, B.H. Waters, J.R. Smith, N. Kuster: Evaluation of wireless resonant power transfer systems with human electromagnetic exposure limits, IEEE Transactions on Electromagnetic Compatibility, vol. 55(2), pp. 265-274 (2013)

8. R. Pinto, V. Lopresto, A. Genovese: Human exposure to wireless power transfer systems: a numerical dosimetric study, Proc. 11th European Conference on Antennas and Propagation (EUCAP), pp. 1-3, Paris, France (2017)

9. International Commission on Non-Ionizing Radiation Protection: Guidelines for limiting exposure to time-varying electric and magnetic fields for low frequencies $(1 \mathrm{~Hz}-100 \mathrm{kHz})$, Health Phys., vol. 99, pp. 818-836 (2010)

10. IEEE Standard for Safety Levels with Respect to Human Exposure to Radio Frequency Electromagnetic Fields, $3 \mathrm{kHz}$ to $300 \mathrm{GHz}$, IEEE Std. C95.1 (2005)

11. I. Laakso, A. Hirata: Evaluation of the induced electric field and compliance procedure for a wireless power transfer system in an electrical vehicle, Phys. Med. Biol., vol. 58, pp. $7583-7593(2013)$

12. F. Wen, X. Huang: Human exposure to electromagnetic fields from parallel wireless power transfer systems, International Journal of Environmental Research and Public Health, vol. 14(2), pp. 1-15 (2017)

13. International Agency for Research on Cancer (IARC), Non-ionizing radiation, part 2: radiofrequency electromagnetic fields, IARC Monograph, vol. 102, Lyon, France (2013)

14. D. Woods: Four concepts for resilience and the implications for the future of resilience engineering, Reliability Engineering and System Safety, vol. 141, pp. 5-9 (2015)

15. S. Sridhar, A. Hahn, M. Govindarasu: Cyber-physical system security for the electric power grid, Proceedings of the IEEE, vol. 100(1), pp. 210-224 (2012)

16. S. Sheard: A framework for system resilience discussions, INCOSE International Symposium, vol. 18(1), pp. 1243-1257 (2008)

17. V. Monteiro, H. Gonçalves, J. C. Ferreira, J. L. Afonso: Batteries charging systems for electric and plug-in hybrid electric vehicles, New Advances in Vehicular Technology and Automotive Engineering, 1st ed., pp. 149-168 (2012)

18. S. Ahn, J. Pak, T. Song, H. Lee, J.-G. Byun, D. Kang, C.-S. Choi, E. Kim, J. Ryu, M. Kim, Y. Cha, Y. Chun, Y., C.-T. Rim, J.-H. Yim, D.-H. Cho, J. Kim: Low frequency electromagnetic field reduction techniques for the on-line electric vehicle (OLEV), IEEE Int. Symposium on Electromagnetic Compatibility, Fort Lauderdale, Florida, USA (2010)

19. M. Kim, S. Kim, Y. Chun, S. Park, S. Ahn: Low frequency electromagnetic compatibility of wirelessly powered electric vehicles, International Symposium on Electromagnetic Compatibility, Tokyo (2014)

20. P. Solín: Partial differential equations and the finite element method, John Wiley and Sons, pp. 269-291 (2006)

21. E.N. Baikova, S.S. Valtchev, R. Melicio, V. Fernão Pires: Wireless Electromagnetic interference impact of wireless power transfer system on data wireless channel, in Technological Innovation for CyberPhysical Systems, Springer, pp. 293-301 (2016)

22. E.N. Baikova, S.S. Valtchev, R. Melicio, V. Fernão Pires: Wireless power transfer impact on data channel, IEEE Int. Symposium on Power Electronics, Electrical Drives, Automation and Motion, pp. 582-587, Capri, Italy (2016) 